from established usage, he has succeeded in giving several subjects a new aspect. As far as possible dead wood has been cut out and live material put in its place. The large number of new and attractive problems drawn from everyday life and from the elements of mensuration and physics will be greatly appreciated by the teacher. Considerable prominence has been given to graphical methods. The author has used them very effectively in treating proportion and variation, in the solution of equations, in establishing the notion of roots and logarithms, and even in explaining interpolation in a logarithmic table. Graphical methods not only make these subjects clearer and more real to the pupil, but will prove of great service to him whether he stops study with the high school or not. In the appendix some of the more advanced topics are treated as permutations and combinations, the binomial theorem, limits and infinite series, irrational and complex numbers.

It needs hardly to be added that the reasoning is sound and in accordance with present-day standards. The pupil will have no vicious habits of thought to eradicate if he goes on with his mathematics. In this connection we may note the emphasis the author lays on checking the solution of equations, a point too often entirely overlooked.

It is a good sign for the future of mathematics in this country when men of the calibre of Professor Hedrick are willing to take time from their own researches to write a work of this character. We wish his little book all success.

\title{
James Pierpont.
}

Die. Lehre von den geometrischen Verwandtschaften. Erster Band: die Verwandtschaften zwischen Gebilden erster Stufe. By Rudolf Sturm. Leipzig and Berlin, B. G. Teubner, 1908. $\mathrm{xii}+415 \mathrm{pp}$.

THE treatise on geometric transformations, of which the present volume is the first part, is estimated to consist of four volumes, the manuscript of which is all in the hands of the printer. The others will be published as soon as possible.

While the first volume is concerned with forms of one dimension, it is not simply a text-book on projective geometry, but considers multiple correspondences, general involutions, trilinearity, and the problem of homography in two and in three dimensions. The first half of the book is concerned with parametric representation, anharmonic ratios, perspectivity, linear 
substitution, duality, involution, and polarity. Much stress is laid on directed elements, and all parametric representations are founded on the anharmonic property. The general method is synthetic, but so much use is made of algebraic substitutions that a long article (seventeen pages) is included, explaining the elements of the theory of invariants. All the steps of every proof are given, and a careful interpretation of each step is made. Indeed it would be easy for a patient student to acquire an accurate and extensive knowledge of many of the chapters of projective geometry by reading this book without a teacher. While the use of anharmonic ratios is by no means so great as was predicted for them a few decades ago, yet in parametric representation, theory of invariants, and theory of linear groups they have a lasting value, and most of these important interpretations are found in this book.

The footnotes give a large number of references, but too frequently these mention only the older literature. The technical words used in the text have become in part obsolete, and frequently have definitions different from those used by other writers. Fortunately the book is provided with an extensive glossary which largely atones for the confusion arising from the use of the older words.

The second half begins with an algebraic treatment of multiple correspondence, similar to that in the first volume of the author's treatise on line geometry, ${ }^{*}$ but much more extensive. Halphen's precise application of Carnot's theorem is made the basis of the derivation of Plücker's formulas, and of the proof of the invariance of the genus under birational transformations.

The chapter on the generation of multiple correspondence is rather too abstract. This most useful tool in the study of curves and ruled surfaces frequently offers great difficulty to the reader, and a systematic exposition of the theory should be made as concrete as possible.

The word involution is used to define the usual involution, as well as symmetric $(n, n)$ correspondences which are not involutional. An instructive example of multiple coincidences is followed by the warning to look out for very serious trouble in more complicated cases.

The existence of various involutions and multiple correspondences on the conic is treated in a synthetic manner, with

* Die Gebilde ersten und zweiten Grades der Liniengeometrie in synthetischer Behandlung, Leipzig, Teubner, 1892 ; vol. 2, 1893 ; vol. 3, 1896. 
the occasional introduction of an equation, which makes the theorems much clearer. In this regard, most of the treatment is less formal but more easily understood than the corresponding part of Reye's treatise.

Since the author holds strictly to the traditions of the Steiner school (though never advocated by Steiner), no figures appear in the book. In many cases the explanations are sufficiently clear without this help, but in discussions of pencils of collineations, products of involutions, and similar subjects, the text could be made much briefer by the addition of appropriate figures.

The fifteen pages devoted to the twisted cubic curve contain a large number of results, skilfully arranged. The chapter on trilinearity is much more systematic than the treatment given this important subject in other places. A number of equations are used to establish the existence of neutral pairs, singular elements, and principal points, but much of the discussion is synthetic. The theory of the cubic surface follows easily from the elementary principles of trilinearity.

Nearly a hundred pages are occupied with the so-called problem of projectivity, $i$. $e$., given a series of arbitrary points $A_{k}$, and their associates $B_{k}$, for any point $P$ to find the corresponding point $Q$ such that the pencil $Q B_{k}$ is projective with $P A_{k}$. The problem has no meaning if the number of arbitrary points is less than four, and becomes impossible when it is greater than seven. In particular, the Cremona transformation of degree five having six distinct base points results from five points $A_{k}$. The corresponding problem in space includes the discussion of the tetrahedral complex, and some surfaces belonging to it.

Some birational transformations are found in space which are believed to be new.

The later volumes are to contain the theory of collineations in space of two and of three dimensions, including the singular or degraded forms, and the theory of Cremona transformations and multiple correspondences. VIRGIL SNyder.

Beiträge zur Theorie der linearen Transformationen als Einleitung in die algebraische Invariantentheorie. By W. SсHEIBNER. Leipzig, B. G. Teubner, 1907. 250 pp.

ThE contents of most of this book appeared in the Sächsische Berichte from 1903 to 1907 . The author explicitly declares 